\title{
A Comparison of Root Mean Square Errors on Skeletonization Methods
}

\author{
Neeti Daryal and Vinod Kumar
}

\begin{abstract}
Vectorization is the most fundamental operation in interpretation of line drawings and document analysis. There are several reasons for converting image vectorization. Vector data is normally created from existing natural source image like photographs, scanned images. Choosing a best vectorization method that suits the needs of the system is very important. In general, good methods must preserve information like line geometry and intersection junction as far as possible. It is also important to analyze the error and find the accuracy of the result with respect to the original data. We have compared Skeletonization by Mathematical Morphology and Voronoi Diagrams with original image for vectorizing images. Root mean squre error is one of the good methods to analysis an error on original Image, Mathematical Morphology and Voronoi Diagrams. Literature about above said methods is also included in this paper.
\end{abstract}

Index Terms-Vectorization, skeletonization, mathematical morphology, voronoi diagram, thinning, zhang-suen's algorithm, holt's staircase removal algorithm, matlab, mean, standard deviation, root mean square error.

\section{INTRODUCTION}

Paper maps have always been of prime tool in the field of mapping. Now a days the digital data is more important than the paper maps. All paper maps need to convert all of the available analog geospatial data to digital form. Although the topological maps can be scanned, they cannot be used directly in a GIS system without processing. All the available commercial raster to vector conversion software are semi-automatic and are based on line tracing algorithms and therefore, require a best method. Thus automatic algorithms are required for faster and reliable conversion of maps. The primary objective of this research is to extract the centerline of the lines on a black and white, and grayscale scanned image using skeletonization by Voronoi Diagrams and Mathematical Morphology and compare the result obtained by using both the algorithms. Shows the results and compares the vectorized image by calculating Root mean square error.

\section{SKeletonization By Mathematical Morphology}

Mathematical Morphology is a method for quantitative analysis of spatial structures that aims at analyzing shapes and forms of an object. A Skeleton is basically the centerline extraction of an object that results from thinning [1].Thinning is used to remove selected foreground pixels from binary

Manuscript received December 9, 2011; revised January 18, 2012.

N. Daryal is with Computer Science and Applications, M.L.N.College Yamuna Nagar.

V. Kumar is with Mathematics, J.V.Jain College Saharnpur, India. images like erosion or opening. Vectorization by Mathematical Morphology using skeletonization is done by thinning [2]. Thinning is version the process of reducing a shape to a simpler that still retains the essential features of the original object [3]. The thinned version of the shape is called the skeleton (centerline)[4]. When the skeleton is centered with respect to the boundaries of the original object, it is referred to as the medial-axis or medial-surface. Thinning can be applied only to binary images. Fig. 1 is the binary image and Fig. 2 is skeleton of binary image.

In this paper we have applied Zhang-Suen's algorithm for thinning because it has been used as a basis for comparison of thinning methods for many years and it is comparatively fast and simple to implement [5].

\section{Algorithm}

1. Preprocessing the image with Stentifords acute angle emphasis preprocessing step.

2. Applying Zhang-Suen's algorithm for thinning.

3. Post processing using Holt's staircase removal algorithm.

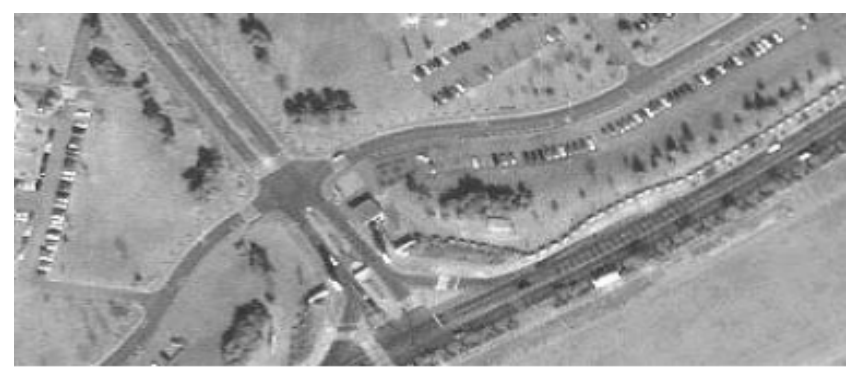

Fig.1. Scanned Satellite Image.

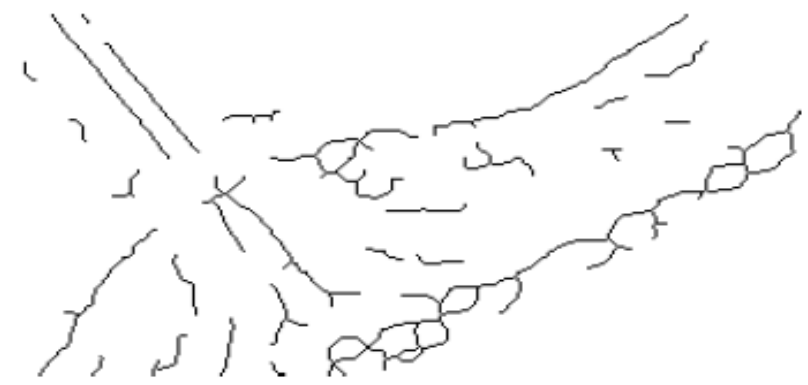

Fig. 2. Skeletonization by Mathematical Morphology.

\section{A. Preprocessing}

Thinning results in necking, tailing and spurious projects in the skeleton. They are called classical thinning artifacts. A narrow point at the intersection of two lines stretched into a small line segment is called necking. Tails are created because of excess thinning, where two lines meet at an acute angle and finally the most common artifact is the creation of 
extra line segments joining a real skeleton which is called a spurious projection, hairs or line fuzz.

Stentiford has suggested a preprocessing step to minimize these thinning artifacts [5]. Since line fuzz is usually caused by some small irregularities in the object outline, a smoothing step before thinning would help remove them. In this research since we pass a median filter to remove noise line fuzz has already been removed. Stentiford suggests a method called acute angle emphasis to deal with necking. In this procedure the pixel near the joint between two lines are set to white if they form an acute angle.

\section{B. Thinning}

Zhang-Suen's algorithm for thinning is a parallel method, meaning that the new value for any pixel can be computed only by using the values obtained from the previous iteration. The algorithm is divided into two sub iterations as shown in Fig 3(a) and Fig 3(b). In one sub iteration, a pixel $I(i, j)$ is deleted (or marked for deletion) if the following four conditions are all true:

1. Its connectivity number is one.

2. It has at least two black neighbors and not more than six.

3. At least one of $I(i, j+1), I(i-1, j)$ and $I(i, j-1)$ are background (white).

4. At least one of $I(i-1, j), I(i+1, j)$ and $I(i, j-1)$ are background.

At the end of this sub iteration the marked pixels are deleted. In the next sub iteration a pixel $I(i, j)$ is deleted (or marked for deletion) if the following four conditions are all true:

1. Its connectivity number is one.

2. It has at least two black neighbors and not more than six.

3. At least one of $I(i-1, j), I(i, j+1)$ and $I(i+1, j)$ are background.

4. At least one of $I(i, j+1), I(i+1, j)$ and $I(i, j-1)$ are background.

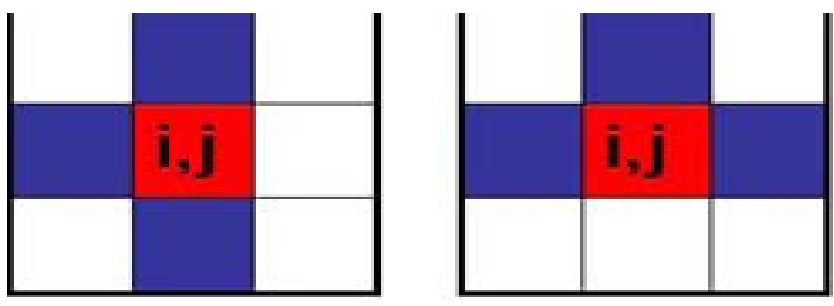

Fig. 3. (a)Pixel marked for deletion in 1st iteration.
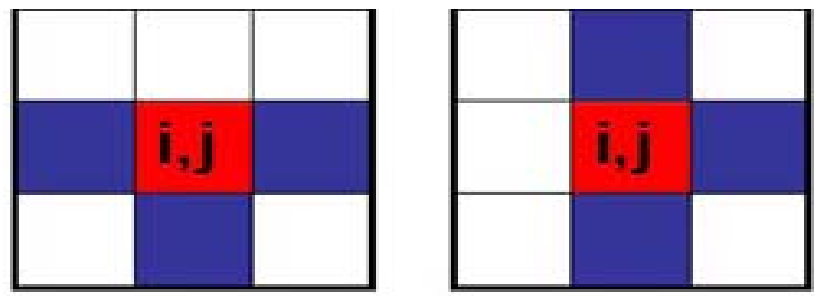

Fig. 3. (b)Pixel marked for deletion in 2nd iteration.

The marked pixels are deleted. If at the end of either sub iteration there are no pixels to be deleted, then the skeleton is complete and the program stops [5].

\section{Post Processing}

In 1987, Holt [5] suggested an improvement to
Zhang-Suen's algorithm that is faster and does not involve sub iterations. The first sub iteration can be written as a logical expression as:

$\mathrm{v}(\mathrm{C})^{\wedge}\left(\sim \operatorname{edge}(\mathrm{C}) \mathrm{V}\left(\mathrm{v}(\mathrm{E})^{\wedge} \mathrm{v}(\mathrm{S})^{\wedge}(\mathrm{v}(\mathrm{N}) \mathrm{V} \mathrm{v}(\mathrm{W}))\right)\right)$

where the center pixel is not marked for deletion in the first sub iteration and the function $\mathrm{V}$ gives a value of the pixel, 1 is true for an object and 0 is false for the background. The edge function is true if $\mathrm{C}$ is on the edge of the object, i.e. having the neighbors between 2 and 6 and connectivity number $=1$. $\mathrm{E}, \mathrm{S}, \mathrm{N}$ and $\mathrm{W}$ correspond to pixels in a particular direction from the center pixel $\mathrm{C}$; $\mathrm{E}$ is east i.e. $\mathrm{I}(\mathrm{i}, \mathrm{j}+1), \mathrm{S}$ is south i.e. I $(i+1, j)$ and so on $[5]$.

The second sub iteration can be written as a logical expression as:

$\mathrm{v}(\mathrm{C})^{\wedge}\left(\sim \operatorname{edge}(\mathrm{C}) \mathrm{V}\left(\mathrm{v}(\mathrm{W})^{\wedge} \mathrm{v}(\mathrm{N}) \wedge(\mathrm{v}(\mathrm{S}) \mathrm{V} \mathrm{v}(\mathrm{E}))\right)\right)$

Holt combined the above 2 expressions with a connectedness preserving condition for parallel execution into the following single expression [5]:

$$
\begin{array}{r}
\mathrm{v}(\mathrm{C})^{\wedge}(\sim \operatorname{edge}(\mathrm{C}) V \\
\left(\operatorname{edge}(\mathrm{E})^{\wedge} \mathrm{v}(\mathrm{N})^{\wedge} \mathrm{v}(\mathrm{S})\right) V \\
\left(\operatorname{edge}(\mathrm{S})^{\wedge} \mathrm{v}(\mathrm{W})^{\wedge} \mathrm{v}(\mathrm{E})\right) V \\
\left(\operatorname{edge}(\mathrm{E})^{\wedge} \operatorname{edge}(\mathrm{SE})^{\wedge} \operatorname{edge}(\mathrm{S})\right)
\end{array}
$$

At times even if thinning is complete, there are some pixels that could be deleted. The idea behind this is that the pixels that form a staircase are removed and we know that half of the pixels that form a staircase can be removed without affecting the shape of the object. So, the center pixel of one of the windows shown in Fig 4 can be deleted.

The center pixel in one of the above can be deleted. In order to avoid creating a new hole, we add a simple condition that one of the values of $X=0$ in Fig 4 . For kernels having a northward bias (Fig 4: a, b) the expression to save a pixel from being deleted in the staircase removal iteration is:

$$
\begin{array}{r}
v(C)^{\wedge} \sim\left(v(N)^{\wedge}\right. \\
\left(\left(v(E)^{\wedge} \sim v(N E)^{\wedge} \sim v(S W)^{\wedge}\left(V v(W)_{-} \sim v(S)\right) V\right.\right. \\
\left.\left.\left.\left(v(W)^{\wedge} \sim v(N W)^{\wedge} \sim v(S E)^{\wedge}(\sim v(E) \bar{V} \sim v(S))\right)\right)\right)\right)
\end{array}
$$

The pass having the southward bias is the same, but with north and south exchanged. However, this method does not remove tails and line fuzz [5].

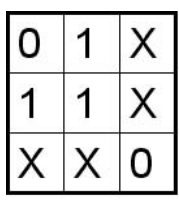

(a)

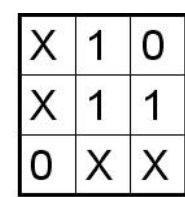

(b)

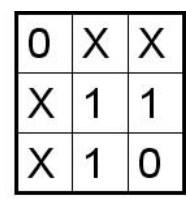

(c)

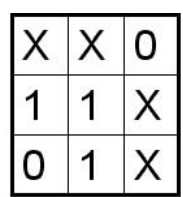

(d)
Fig. 4. Holts Staircase Problem.

\section{SKELETONIZATION BY VORONOI DiAgRAMS}

This approach is based on image processing techniques to extract the basic spatial features from raster data[6]. Let $\mathrm{O}$ be a set of sites in Euclidean space of dimension n. For each site 
o of $\mathrm{O}$, the Voronoi cell $\mathrm{V}(\mathrm{o})$ of $\mathrm{o}$ is the set of points that are closer to o than to other sites of $\mathrm{O}$. The Voronoi diagram $\mathrm{V}$ (O) is the space partition induced by Voronoi cells [7]. An example of the Voronoi Diagram is shown in Fig. 5.

Once the topological properties of the diagram are known, its geometrical properties like coordinates, lengths, angles, etc. can be computed in linear time depending on the number of sites. The computation of Voronoi Diagram can be greatly simplified by working with its dual, which is known as the Delaunay triangulation of the given sites [8]. This allows a cleaner separation between the topological and geometrical aspects of the problem. Voronoi Diagram of a set of generating points can be simply drawn on a piece of paper. However, the drawn diagram or a set of numerical data equivalent to the drawn diagram does not convey enough information for many applications [9]. Assume that a computer program gives a list of line segments corresponding to the Voronoi edges as output. It is easy to draw the diagram using these edges but it is not easy to retrieve information such as a set of Voronoi polygon adjacent to one Voronoi polygon etc. Thus just drawing the Voronoi Diagram is not enough what we need is a data structure from which we can extract a variety of information necessary for applications.

In this research we have chosen to use the quad edge data structure that was developed by Guibas and Stolfi as a primitive topological structure for the representation of any subdivision on a two dimensional manifold [8].

\section{Algorithm}

1. The paper image is scanned and median filter is used to remove [10].

2. Otsu method for thresholding is applied to create binary image.

3. Edge detection operation is applied on the binary image and every pixel on the edge is used as a data point.

4. Incremental Method [11] is used to generate Voronoi Diagram.

5. Then skeleton is extracted from the Voronoi Diagram.

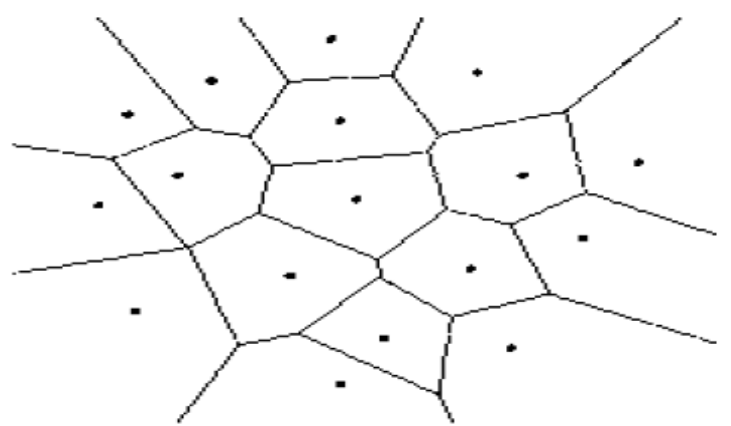

Fig. 5. Voronoi Diagram.

\section{ERror ANALYSIS OF SKELETONIZATION METHODS WITH ORIGINAL DATA}

The main objective of the research is the comparison of Skeletonization using Mathematical Morphology and Voronoi Diagrams. In our case, we have done the first step of vectorizing, i.e. conversion of raster data to vector data using Skeletonization by Mathematical Morphology and Voronoi Diagrams[12]. Mean and standard deviation is applied on the data to analyze and draw meaningful conclusions.Our main objective now is to find the accuracy or the deviation of the vectorized data with the original data. In order to do this we took a scanned image of the portion of Yamuna Nagar map and original data is collected.

The scanned images are shown in Fig. 6, skeletonization using Mathematical Morphology in Fig. 7 and skeletonization using Voronoi Diagram in Fig. 8. The result of Skeletonization was imported into MATLAB and was geo-referenced and projected.

\section{RESUlT AND CONCLUSION}

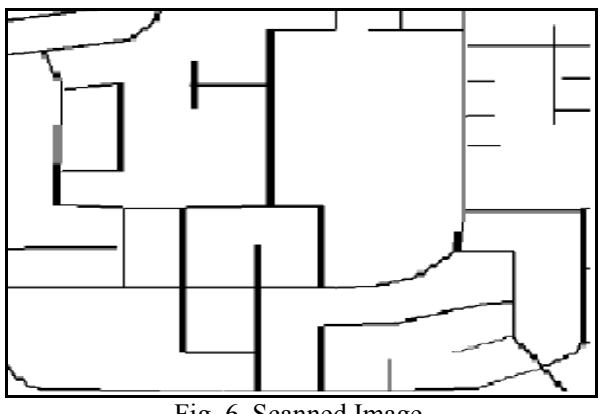

Fig. 6. Scanned Image.

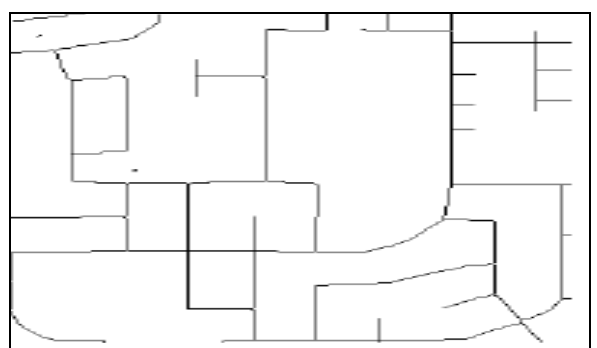

Fig. 7. Skeletonization by Mathematical Morphology.

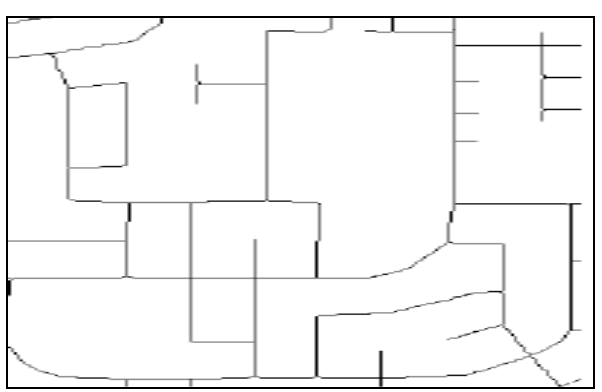

Fig. 8. Skeletonization by Voronoi Diagrams.

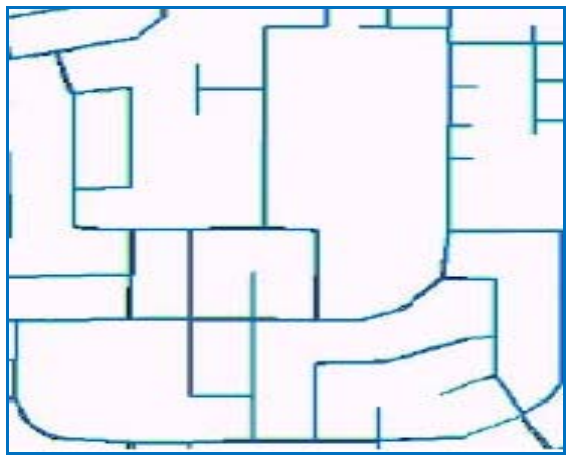

Fig. 9. Overlay Image.

Manual digitizing is tedious and time consuming. In this method the accuracy of maintaining the geometry of the 
object and digitizing in the center of the object completely depends on the operator. For best results the images were overlayed on the original data. The result of overlay of skeletonization by Mathematical Morphology over the original image is shown in Fig. 9. After overlaying the image it was found that manual digitization had an error of upto 1.5 meters. The result produced by Mathematical Morphology had an error of upto 2 meters and the result generated by Voronoi Diagrams had an error of upto 4 meters. These errors were measured using the measuring tool in MATLAB. It was also found that the error was not continuous and in most of the locations it matched completely.

Then twenty well distributed matching points were selected and their respective coordinates were saved in a text file using MATLAB. Using the original true coordinates and the coordinates collected from the two methods the mean and standard deviation was calculated using the following formula [13]:

$$
\begin{gathered}
\mu_{d x}=\sum_{\substack{i=1 \\
n}}^{n}\left(x_{i}-x_{\text {true }}\right) \\
\mu_{d y}=\sum_{i=1}\left(y_{i}-y_{\text {true }}\right) \\
\sigma_{d x}=\operatorname{sqrt}\left(\sum_{i=1}^{n}\left(x_{i}-x_{\text {true }}\right)^{2}\right) \\
\sigma_{d y}=\operatorname{sqrt}\left(\sum_{i=1}^{n}\left(y_{i}-y_{\text {true }}\right)^{2}\right)
\end{gathered}
$$

where $\mu_{\mathrm{dx}}$ is the mean of the difference between the $\mathrm{x}$ coordinates and $\mu_{\mathrm{dy}}$ is the mean of the difference between the y coordinates. Similarly $\sigma_{\mathrm{dx}}$ and $\sigma_{\mathrm{dy}}$ are the standard deviation of $x$ and coordinates from the true values i.e., the estimated error or accuracy in the $\mathrm{x}$ and $\mathrm{y}$ direction respectively. In (5) and (6) the reason for using dividing by $n$ and not $n-1$ is that we are using the true value of $\mathrm{x}$. The Root Mean Square error (RMSE) represents the difference between the original control points and the new control point locations calculated by the transformation process. The transformation scale indicates how much the image being digitized will be scaled to match the real-world coordinates [14]. In our case we will consider the well distributed 20 coordinates that we called from the original image and the vectorized image using the three methods. In most of the cases Root Mean Square Error(RMSE) is considered synonymous to standard deviation. RMSE can be calculated by using the following formula:

$$
\begin{gathered}
\operatorname{RMSE}_{\mu}=\operatorname{sqrt}\left(\mu_{d x}^{2}+\mu_{d y}^{2}\right) \\
R M S E_{\sigma}=\operatorname{sqrt}\left(\sigma_{d x}^{2}+\sigma_{d y}^{2}\right)
\end{gathered}
$$

\begin{tabular}{|c|c|c|c|c|c|c|}
\hline & \multicolumn{2}{|c|}{ Scanned Image } & \multicolumn{2}{|c|}{$\begin{array}{c}\text { Skeletonization by } \\
\text { Mathematical } \\
\text { Morphology }\end{array}$} & \multicolumn{2}{|c|}{$\begin{array}{c}\text { Skeletonization } \\
\text { by Voronoi } \\
\text { Diagram }\end{array}$} \\
\hline & $\begin{array}{c}\text { Standard } \\
\text { Deviation }\end{array}$ & Mean & $\begin{array}{c}\text { Standard } \\
\text { Deviation }\end{array}$ & Mean & $\begin{array}{c}\text { Standard } \\
\text { Deviation }\end{array}$ & $\begin{array}{c}\text { Mea } \\
\mathrm{n}\end{array}$ \\
\hline $\mathrm{X}$ & 0.3615 & 0.1059 & 0.7559 & $\begin{array}{c}0.285 \\
7\end{array}$ & 0.9047 & $\begin{array}{c}0.41 \\
02\end{array}$ \\
\hline $\mathrm{Y}$ & 0.6203 & 0.1286 & 1.1339 & $\begin{array}{c}-0.42 \\
86\end{array}$ & 1.3305 & $\begin{array}{r}-0.6 \\
176\end{array}$ \\
\hline RMSE & 0.7179 & 0.1665 & 1.3628 & $\begin{array}{c}0.515 \\
1\end{array}$ & 1.6089 & $\begin{array}{c}0.74 \\
14\end{array}$ \\
\hline
\end{tabular}

The mean and standard deviation of three methods for the first and second images are shown in TABLE I:
TABLE I: MEAN, STANDAR DEVIATION AND RMSE

From TABLE I we can infer that in our case best accuracy is of Skeletonization by Mathematical Morphology as compare to the Skeletonization by Voronoi Diagrams.

\section{REFERENCES}

[1] E. S. Deutsch, "Thinning algorithms on rectangular, hexagonal and triangular arrays," Communications of the ACM, vol. 15, pp. 827-837, 1972 .

[2] E. R. Davies and A. P. N. Plummer, "Thinning algorithms: a critique and a new methodology," Pattern Recognition, vol.14, pp 53-53, 1981

[3] J. Serra, "Image Analysis and Mathematical Morphology," I, II Academic Press, 1982.

[4] H. Tamura, "A Comparison of line thinning algorithms from digital geometry viewpoint," In Proc. of the 4th International Conference on Pattern Recognition, Kyoto, Japan, pp.715-719, 1978.

[5] L. Boatto, et al,"An Interpretation System for Land Register Images," IEEE Computer, vol. 25, no. 7, pp. 25-32, 1991.

[6] R. W. Smith, “Computer processing of line images: A survey," Pattern Recognition x, vol. 20, no. 1, pp. 7-15, 1978.

[7] R. Kasturi, S. T. Bow, W. El-Masri, J. Shah, J. R. Gattiker, and U. B. Mokate, "A System for Interpretation of Line Drawings," IEEE Transactions on Pattern Analysis and Machine Intelligence, vol. 12, no. 10, pp. 978-992, 1990.

[8] W. K. Nicholson, "Elementry Linear Algebra with Applications," Prindle, Weber \& Schmidt, 1986

[9] W. Liu and D. Dori, "From raster to vectors: Extracting visual information from line drawings," Pattern Analysis \& Applications, vol. 2, pp.1021, 1999.

[10] S. D. Zenzo and A. Morelli, "A useful image representation," In Proc. of the 5th International Conference on Image Analysis and Processing, Word Scientific Publishing, Singapore, pp.170-178, 1989.

[11] L. T. Watson, K. Arvind, R. W. Ehrich, and R. M. Haralick, "Extraction of lines and regions from greytone line drawing images," Pattern Recognition, vol.17, pp. 93-507, 1984.

[12] L. Lam, S. W. Lee, and C. Y. Suen, "Thinning methodologies - A comprehensive survey," IEEE Transactions on Pattern Analysis and Machine Intelligence, vol. 14, no. 9, pp. 869-887, 1994.

[13] R. C. Gonzalez, R. E. Woods, L. Steven, and Eddins, "Digital Image Processing using MATLAB," Pearson Prentice hall, Pearson Education In, 2007

[14] C. M. Eastman, "1990Vector versus raster. A functional comparison of drawings technologies," In Proc IEEE Computer Graphics and Applications, pp.68-80, 1990.

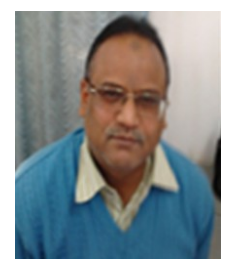

Vinod Kumar born on 09-05-1961. He passed his B.Sc. in 1981 and M.Sc. in Mathematics in 1984 from Meerut University Meerut. He obtained his $\mathrm{Ph} . \mathrm{D}$ degree in Mathematics from university of Roorkee, Roorkee in 1994 in the area of 'Plasma Physics, fluid dynamics and numerical analysis'. Now he is working as a Associate Professor and Dean in J V Jain college, Saharanpur UP, India. 
Neeti Daryal born on $31^{\text {st }}$ March,1975. She received the B.Sc. degree in Computer Science from Kurukshetra University ,Kurukshertra in 1996, M.Sc. degree in Information Technology in 1999 and MCA degree in the year 2004. Now she is pursuing her Ph.D Degree in Image Processing. She joined as Lecture in the Department of Computer Science and Applications in 2000, where she is currently an Assistant Professor and Head of the Department. The research interest of Neeti Daryal includes Image Processing, Satellite Imageries and Pattern Recognition. 